\title{
Taux de reproduction du puceron du pois (Acyrthosiphon pisum Harris) et teneur en acide médicagénique de la luzerne (Medicago sativa $L$ )
}

\author{
R Bournoville \\ Laboratoire de zoologie, Inra, F-86600 Lusignan, France
}

(Reçu le 22 octobre 1993; accepté le 12 janvier 1996)

\begin{abstract}
Résumé - Une douzaine de clones et d'hybrides simples de luzerne ont servi à comparer les capacités de multiplication du puceron du pois aux résultats de tests biologiques et d'analyses chimiques permettant de doser une fraction active des saponines de la luzerne, l'acide médicagénique. Les analyses chimiques font appel à la chromatographie liquide haute performance. Les méthodes biologiques se réfèrent à la quantité d'aliment sec métabolisé par la larve du coléoptère Tenebrio molitor $L$ (Tenebrionidae), et à l'inhibition du développement des colonies du champignon Trichoderma viride Pers. Des larves de quatrième stade du puceron du pois ont été élevées en conditions contrôlées (température $20^{\circ} \mathrm{C}$, photophase 16 heures) dans des cagettes installées à l'extrémité des tiges des luzernes poussant dans des caissettes. Les taux nets de reproduction de ces aphides établis après 14 jours d'expérimentation ne sont pas corrélés aux résultats des tests biologiques ou du dosage chimique de l'acide médicagénique, qui sont pour leur part corrélés entre eux. On en déduit que l'acide médicagénique n'est pas une fraction active impliquée dans les mécanismes de résistance de la luzerne au puceron du pois.
\end{abstract}

facteur antinutritionnel / aphide / test biologique / analyse chimique / résistance aux déprédateurs

Summary - Net reproductive rate of the pea aphid (Acyrthosiphon pisum Harris) and medicagenic acid content of lucerne. Medicagenic acid, a major component of the saponin content of lucerne, has been reported by some authors as one of the possible mechanisms of resistance of lucerne to the pea aphid. We have measured the net reproductive rate of the pea aphid on 12 clones or hybrids of lucerne, derived from cultivars or lines of American (La, Res, $T e)$ and French $(12,58$, Eur) origins. The pea aphid performances were tested on plants growing under controlled conditions (temperature $20{ }^{\circ} \mathrm{C}$, photophasis $16 \mathrm{~h}$ ). Before the budding stage of the plants, small cages were placed at the tips of the stems and infested with five fourth-stage pea aphid larvae. Survival and fecondity of these insects were evaluated after 7 and 14 days. Dried powdered aerial parts of the 12 groups of plants were used for chemical and biological tests. High performance liquid chromatography was used to measure the medicagenic acid content. Two kinds of biological tests were carried out to assess the medicagenic acid contents. The 'yellow mealworm test' used larvae of Tenebrio molitor and quantified the dry food assimilated; the Trichoderma viride test is a traditional biological test which estimates the growth inhibition of fungus. The reproductive rates of the pea aphids were not correlated with the medicagenic acid content of the plants, as evaluated by the biological tests or the chemical analysis. We conclude that medicagenic acid is not a factor in the resistance of lucerne to the pea aphid.

medicagenic acid / pest resistance / biological test / aphid / allelochemical compound 


\section{INTRODUCTION}

Dans sa mise au point sur la résistance des plantes aux aphides, Auclair (1989) rappelle que, malgré les nombreux programmes entrepris en Amérique du Nord pour la sélection de cultivars de luzerne résistants au puceron du pois, on est peu renseigné sur les causes de cette résistance. Parmi les éléments publiés, il rapporte l'influence possible de deux catégories majeures de substances, les nutriments et les composés antinutritionnels. Parmi les premiers, le rôle des sucres et des acides aminés a fait l'objet d'études récentes. Celles-ci ont porté sur l'analyse de la sève phloémienne obtenue par exsudation artificielle (Febvay et al, 1988), ou par stylectomie (Girousse et Bournoville, 1994) de clones de luzerne qui diffèrent pour les capacités de multiplication du puceron du pois. Les recherches ont également mis en œuvre des milieux holidiques reproduisant la composition de cette sève (Rahbé et al, 1988). Les résultats de ces derniers travaux prouvent que, lorsque la composition en nutriments du régime artificiel copie celle de la sève de deux génotypes de luzerne, l'un sensible, l'autre résistant au puceron, on ne fournit pas une explication complète de la résistance.

Quant aux composés antinutritionnels, les résultats, d'ailleurs assez variables, se rapportent à la teneur en saponines. Ces hétérosides, fréquents dans le monde végétal, sont constitués d'une aglycone (la sapogénine), liée à un ou plusieurs glucides. Parmi les diverses aglycones trouvées chez la luzerne, celles de type «acide médicagénique" présentent, en raison de leur fonction carboxylique, une importante activité sur les animaux: des effets sur les taux de croissance sont connus lorsqu'on incorpore de la farine de luzerne à la ration de vertébrés monogastriques (Monties, 1981). Dans le cadre de la mise en œuvre d'un programme de sélection de cultivars de luzerne pour une faible teneur en saponines, nous avons montré (Bournoville, 1993), tant en conditions contrôlées qu'au champ, que la teneur globale de variétés de luzerne en saponines ne détermine pas le niveau de résistance de la luzerne au puceron du pois. Le présent travail complète le précédent, en s'intéressant à la relation existant entre $A$ pisum et la teneur en acide médicagénique, fraction réputée toxique pour cet aphide, lorsqu'elle est incorporée dans la composition de régimes artificiels servant à l'alimenter (Horber et al, 1974 ; Krzymanska et Waligora, 1986). Pour cela, nous avons détermi- né les capacités de multiplication du puceron du pois sur une douzaine de clones ou d'hybrides simples dont nous avons également déterminé la teneur en acide médicagénique par des tests biologiques ou des analyses chimiques. Jusqu'à une date récente, la séparation, la purification et le dosage chimique de saponines de la luzerne étaient difficiles. Dans ses articles consacrés à l'activité des saponines de la luzerne sur les larves du coléoptère Tenebrio militor $\mathrm{L}$, Pracros (1988a, b) signale l'intérêt de tests biologiques révélateurs de la teneur en saponines et plus particulièrement de certaines fractions actives, dont l'acide médicagénique.

\section{MATÉRIEL ET MÉTHODES}

\section{Les plantes}

Nous avons utilisé des clones ou des hybrides simples, issus de leurs croisements, pour réaliser l'ensemble des tests. Les clones provenaient chacun d'une seule plante vigoureuse des pépinières du Centre de recherches de Lusignan (Vienne). Leurs origines géographiques sont nord-américaines (clones La $=$ Lahontan, $T=$ Team, $\mathrm{R}=$ Resistador) ou françaises (clones 12, 58, $E=$ Europe). Pour les tests relatifs au puceron, les clones et les hybrides étaient installés en chambre de culture et poussaient dans des caissettes de bois $(\mathrm{L}=40 \mathrm{~cm}, l=15 \mathrm{~cm}, \mathrm{~h}=15 \mathrm{~cm})$ contenant un mélange standardisé d'un tiers de terre franche, un tiers de sable, un tiers de terreau. Ce dispositif ne permettait pas d'obtenir la matière sèche nécessaire aux autres analyses. C'est pourquoi les clones et les hybrides ont été multipliés et placés en pépinière sur le site du Centre de recherches de Lusignan. On a récolté la matière verte aérienne de ces plantes avant le début de la floraison. Chaque lot de fourrage a été séché à l'étuve à $80^{\circ} \mathrm{C}$ puis finement broyé (maille de tamis de $1 \mathrm{~mm}$ ). La poudre obtenue a servi aux analyses chimiques et aux tests biologiques. Seuls les résultats des génotypes ayant fourni une biomasse en quantité suffisante pour la réalisation de l'ensemble des tests, soit une douzaine, sont ici exploités (tableau I).

\section{Les pucerons et l'estimation du taux net de reproduction}

À partir d'un seul individu virginopare aptère d'A pisum recueilli dans une luzernière à Lusignan, on a multiplié en pièce climatisée (température : $20^{\circ} \mathrm{C} \pm 2$, photophase : 16 heures) un clone du puceron sur un cultivar sensible (Europe) de luzerne. À intervalles réguliers, on a prélevé dans cette souche des larves de $4^{\mathrm{e}}$ stade dépourvues d'ébauches alaires, ce qui permet de s'as- 
Tableau I. Valeurs des génotypes de luzerne dans les différents tests biologiques et chimiques.

$\begin{array}{ccccc}\text { Génotype } & \text { Puceron } & \text { Tenebrio } & \text { Trichoderma } & \text { Acide médicagénique } \\ & \text { Taux net de reproduction } & \text { Aliment sec métabolisé } & \text { (\% de développement } & \text { (\% de matière sèche) } \\ \text { après 14 jours pour cinq individus } & \text { (mg/larve) } & \text { par rapport au témoin) } & \end{array}$

$\begin{array}{lrrr}58 \times \mathrm{T} & 464 & (16) & 14,2 \\ 12 \times \mathrm{E} & 395 & (18) & 21,6 \\ \text { Res } \times \mathrm{E} & 361 & (15) & 12,2 \\ \text { Res } \times \text { La } & 346 & (29) & 46,6 \\ 12 \times \mathrm{La} & 302 & (31) & 30,0 \\ 58 \times \mathrm{La} & 278 & (23) & 41,0 \\ \mathrm{E} & 244 & (17) & 13,2 \\ 12 \times \mathrm{T} & 225 & (20) & 9,6 \\ 12 & 146 & (38) & 3,7 \\ \mathrm{E} \times \mathrm{La} & 139 & (23) & 25,6 \\ \mathrm{La} & 56 & (17) & 25,9 \\ \text { T } \times \text { La } & 43 & (16) & 33,2\end{array}$

$\begin{array}{ll}(0,9) & 46,0 \\ (1,1) & 31,7 \\ (0,9) & 38,4 \\ (1,6) & 46,1 \\ (1,6) & 47,0 \\ (0,9) & 57,4 \\ (0,8) & 38,4 \\ (0,5) & 37,5 \\ (0,7) & 22,2 \\ (1,1) & 51,9 \\ (0,5) & 40,8 \\ (1,3) & 61,7\end{array}$

$\begin{array}{ll}(1,8) & 0,025 \\ (1,6) & 0,021 \\ (1,6) & 0,019 \\ (0,9) & 0,009 \\ (2,1) & 0,014 \\ (1,4) & 0,011 \\ (1,0) & 0,037 \\ (1,3) & 0,028 \\ (1,9) & 0,027 \\ (0,8) & 0,009 \\ (0,8) & 0,013 \\ (0,9) & 0,007\end{array}$

Les valeurs moyennes sont suivies de l'erreur standard.

surer qu'en se développant ces individus donneront des virginopares aptères. On a déposé ces larves, par groupe de 5 , dans des cagettes $(20 \mathrm{~cm} \times 12 \mathrm{~cm} \times$ $12 \mathrm{~cm}$ ) installées à l'extrémité des tiges de luzerne que I'on souhaite tester, selon le dispositif décrit par Bournoville et Cantot (1974), à raison de 10 répétitions par génotype. Les plantes poussent en chambre de culture (température : $20{ }^{\circ} \mathrm{C} \pm 2$, photophase : 16 heures). Quant aux tiges où sont installées les cagettes, leur phénologie varie du stade végétatif à l'apparition du premier niveau de boutons floraux. Après 7 jours d'élevage, les pucerons survivants devenus adultes dans l'intervalle sont recueillis, leurs descendance est comptée et supprimée. On réinstalle les groupes d'adultes dans les mêmes conditions dans des cagettes sur de nouvelles tiges. Sept jours après (soit le $14^{e}$ jour après le dépôt initial des larves de quatrième stade), l'expérience est arrêtée, après que la survie et la fécondité des adultes ont de nouveau été évaluées. Le taux net de reproduction des individus initiaux (nombre total de descendants obtenus pour cinq larves de quatrième stade initiales) est le critère de classement de la valeur des génotypes, tel que rapporté par Bournoville (1978).

\section{Les tests biologiques et le dosage chimique de l'acide médicagénique}

Le test Tenebrio, décrit par Pracros (1988a), se réfère au poids sec d'aliment métabolisé (c'est-à-dire la quantité d'aliment sec fourni diminué de la quantité d'aliment sec non consommé et de la quantité de fèces produites par les insectes) par cinq lots de 20 jeunes larves du ver de farine par génotype, élevées pendant 4 semaines sur les divers régimes dans une enceinte climatisée $\left(25{ }^{\circ} \mathrm{C}, 80 \%\right.$ d'humidité relative). Le régime est constitué de $20 \mathrm{~g}$ de poudre sèche des parties aériennes des luzernes, additionnés de $80 \mathrm{~g}$ de glucose, de $4 \mathrm{~g}$ d'une mélange de sels minéraux adaptés aux besoins de l'insecte (formulation de MacCollum), de $10 \mathrm{~mL}$ d'une solution de vitamines du groupe $B$ et de $10 \mathrm{~mL}$ d'eau distillée.

Le test Trichoderma, décrit par Pietraszek (1985) selon la technique de Zimmer et al (1967), consiste à mesurer la croissance du champignon $T$ viride, durant 48 heures à $28^{\circ} \mathrm{C}$, selon deux axes perpendiculaires, exprimée pour chaque lot de poudre de luzerne en pourcentage du diamètre d'un témoin dépourvu de composé antinutritionnel, à raison de six répétitions par génotype.

Les analyses chimiques, décrites par Lila et al (1989) selon les méthodes de Massiot et al (1988), ont permis d'obtenir la teneur en acide médicagénique par chromatographie liquide haute performance en phase inverse méthanol-eau, après extraction des saponines brutes et isolement des génines. Une seule analyse a été réalisée par génotype, à partir de $15 \mathrm{~g}$ de poudre de matière sèche.

\section{RÉSULTATS}

Les taux nets de reproduction du puceron du pois déterminés au cours de 2 semaines d'élevage sur les différentes plantes varient dans de fortes proportions (tableau I). Le facteur de variation entre les extrêmes est de l'ordre de 10 . II est principalement le reflet des mortalités des adultes au cours des deux semaines de l'expérience. Ces variations peuvent paraître élevées. 
Elles relèvent sans doute du fait que nous avons utilisé des clones de plantes et quelques hybrides simples, c'est-à-dire des plantes à génotype bien différencié par rapport aux cultivars qui, chez la luzerne, constituent sur le plan génétique des populations. Ces faits interdisent tout rapprochement avec des tests portant sur des cultivars. Par ailleurs, les conditions expérimentales de nos essais qui incluaient cinq larves de quatrième stade par cagette constituent une autre source de variabilité des résultats en raison des densités de descendants obtenus.

Les valeurs moyennes des poids secs métabolisés par les larves du ver de farine s'étendent de 3,7 à $46,6 \mathrm{mg}$ dans les résultats du tableau $\mathrm{I}$. Elles sont plus faibles que celles signalées par Pracros (1988a), qui mentionne pour sa part des valeurs variant de 21,8 à $66,1 \mathrm{mg}$ par larve dans des expériences utilisant également les parties aériennes de cultivars de luzernes. L'inhibition du développement de $T$ viride se situe dans nos résultats dans un intervalle de variation de 22 à $62 \%$ par rapport au lot témoin. Pour des analyses portant également sur les parties aériennes de cultivars de luzerne au stade de début de floraison, Pietraszek (1985) indique des gammes des résultats de tests Trichoderma de cet ordre de grandeur. Quant aux analyses chimiques, la teneur de nos plantes en acide médicagénique varie de 0,007 à $0,037 \%$. Elle est plus faible que celle publiée par Pracros (1988b) qui s'étend de 0,011 à $0,1 \%$. Cependant, cet auteur a déterminé la teneur de feuilles desséchées de cultivars de luzerne, tandis que nous avons réalisé nos analyses sur l'ensemble des parties aériennes des plantes.

Lila et Furstoss (1986) signalent que le facteur explicatif le plus significatif de l'inhibition de croissance de Tenebrio et de Trichoderma est la teneur en acide médicagénique. Pracros (1988b) rapporte également que l'acide médicagénique est un élément essentiel de l'effet antinutritionnel de la luzerne envers $T$ molitor. Dans le tableau II, les coefficients de corrélation calculés entre les diverses variables que nous avons étudiées suggèrent que le taux de multiplication du puceron du pois n'est pas significativement lié $(p=0,05)$ aux estimations biologiques de la teneur des plantes en acide médicagénique, ni à la teneur de nos luzernes en ce composé, alors que les autres valeurs le sont entre elles. Avec 57 échantillons, Lila et Furstoss (1986) obtiennent un coefficient de corrélation de 0,82 , en valeur absolue, liant le test Trichoderma et la teneur en acide médicagénique et de 0,55 (avec 44 échantillons), pour la liaison entre Tenebrio et acide médicagénique. Sur 41 échantillons la corrélation entre le poids d'aliment métabolisé par Tenebrio et la teneur en acide médicagénique est de $-0,73$ selon Pracros (1988b).

\section{DISCUSSION ET CONCLUSION}

Une première remarque portera sur les méthodologies utilisées dans nos expériences. On peut s'interroger sur l'effet de la mise en place dans des conditions variables des plantes ayant servi à l'ensemble de nos tests. Dans leur étude sur la teneur de variétés de luzerne en saponines, Lila et al (1989) signalent que les effets génotypiques sont déterminants par rapport aux conditions de pousse des plantes.

Notre expérimentation ne met pas en évidence de corrélation significative entre la teneur en acide médicagénique et le taux de reproduction du puceron du pois. Ce résultat diffère des élements publiés sur l'incorporation de fractions de saponines à des régimes artificiels du puceron du pois. Horber et al (1974), après avoir séparé 10 fractions d'aglycones des saponines de deux cultivars de luzerne, ont incorporé ces diverses fractions à la dose de $0,1 \%$ dans un régime artificiel, d'ailleurs peu adapté aux larves du puceron du pois, quant à sa teneur en saccharose. Ils constatent que les extraits du cultivar Du Puits,

Tableau II. Matrice des coefficients de corrélation entre les variables du tableau I.

\begin{tabular}{lcccc} 
& Puceron (taux net de reproduction) & Tenebrio & Trichoderma & Acide médicagénique \\
\cline { 3 - 3 } & 1 & & & \\
Tenebrio & $-0,003$ & 1 & 1 \\
Trichoderma & $-0,17$ & $+0,72$ & $-0,68$ & 1 \\
Acide médicagénique & $+0,27$ & $-0,79$ &
\end{tabular}


riches en acide médicagénique, sont plus toxiques que ceux du cultivar Lahontan qui en sont dépourvus. Pour leur part, Krzymanska et Waligora (1986) ont étudié le rôle des saponines extraites de diverses variétés de luzerne sur le puceron du pois et incorporées à la dose de $0,5 \mathrm{mg}$ par $\mathrm{ml}$ de milieu artificiel. Ce sont les jeunes larves qui sont les plus sensibles aux saponines, puisqu'elles meurent en moins de 8 jours. Par ailleurs, ces auteurs ont trouvé que la teneur en acide médicagénique des variétés nord-américaines Washoe et Dawson est importante (de l'ordre de $60 \%$ du total des sapogénines), et suggèrent l'influence de ce composé dans leur classement parmi des cultivars résistants au puceron du pois, sans cependant en démontrer clairement l'effet biologique.

La différence de nos résultats par rapport aux travaux fondés sur l'incorporation des fractions actives de saponines à des régimes artificiels soulève deux questions. La première, sur laquelle on n'est pas renseigné à notre connaissance, est le transport de ces composés dans la sève phloémienne. Certains composés issus du métabolisme secondaire de la plante, comme les alcaloïdes, le sont (Wink, 1984), mais qu'en est-il des génines et plus particulièrement de l'acide médicagénique ? La seconde question qui ne peut que suivre la réponse à la précédente serait la concentration de cette génine dans les conditions naturelles : les tests réalisés avec les régimes artificiels ne biaisent-ils pas la réponse du puceron, par la mise en œuvre d'une concentration trop élevée?

En élevant le puceron sur des plantes entières, nous confirmons en ce qui concerne la teneur en acide médicagénique des luzernes que nous avons testées ce que nous avons trouvé à propos de la teneur totale en saponines (Bournoville, 1993), à savoir que la résistance des génotypes de luzerne vis-à-vis du puceron du pois n'apparaît pas liée à ce composé. En utilisant d'autres plantes de luzerne, nous avons récemment montré (Girousse et Bournoville, 1994) qu'un moindre écoulement de la sève phloémienne après perforation des tubes criblés par des sylets aphidiens entraîne, chez un génotype résistant, une disponibilité alimentaire plus difficile pour les aphides que chez une plante sensible. II ne s'agit donc plus, dans ce cas, de mettre en cause des nutriments ou des composés antinutritionnels dans la relation du puceron avec son hôte, mais l'accessibilité au site alimentaire.

\section{REMERCIEMENTS}

Nous remercions nos collègues de l'Inra pour les tests et les analyses qu'ils ont eu l'amabilité d'effectuer : Mme Pracros de la station de zoologie du Centre de recherche de Bordeaux («Tenebrio»), M Pietraszek ("Trichoderma") et M Furtoss de la station d'amélioration des plantes fourragères du Centre PoitouCharentes (“Acide médicagénique»).

\section{RÉFÉRENCES}

Auclair JL (1989) Host Plant Resistance. In: Aphids, their Biology, Natural Enemies and Control (AK Minks, P Harrenwijn, eds), Elsevier Sc Publ, Amsterdam, vol C, 225-265

Bournoville R (1978) Puceron du pois, Acyrthosiphon pisum Harris. In: Données utiles au sélectionneur pour améliorer la résistance des luzernes à l'égard des maladies et ravageurs (M Massenot, ed), étude INRA-SEI 64, 39-41

Bournoville R (1993) Relation entre le puceron du pois et la teneur en saponines de cultivars de luzerne. Fourrages 136, 547-554

Bournoville R, Cantot P (1974) Matériel utilisé pour l'élevage d'insectes sur la luzerne. Ann Zool Ecol Anim 6, 333-336

Febvay G, Bonnin J, Rahbé Y, Bournoville R, Delrot S, Bonnemain JL (1988) Resistance of different lucerne cultivars to the pea aphid Acyrthosiphon pisum: influence of phloem composition on aphid fecundity. Entomol Exp Appl 48, 127-134

Girousse C, Bournoville R (1994) Role of phloem sap quality and exudation characteristics on performance of pea aphid grown on lucerne genotypes. Entomol Exp Appl 70, 227-235

Horber E, Leath KT, Berrang B, Marcarian V, Hanson $\mathrm{CH}$ (1974) Biological activities of saponin components from Du Puits and Lahontan alfalfa. Entomol Exp App/ 17, 410-424

Krzymanska J, Waligora D (1986) Étude des saponines de la luzerne et de leur action sur le puceron du pois (Acyrthosiphon pisum) (en polonais). Mater XXVI Sess Nauk Inst Ochr Ros/ 267-274

Lila M, Furstoss V (1986) Test rapide de l'activité antinutritionnelle de la luzerne par spectroscopie proche infrarouge. Int Meeting Eucarpia, group Medicago sativa, Pleven (Bulgaria), 134-144

Lila M, Génier G, Pietraszek W (1989) Étude d'un facteur antinutritionnel de la luzerne: les saponines. 16th Int grassland Congress (Nice) II, 779-780

Massiot G, Lavaud C, Le Men-Olivier L, Van Binst G, Miller SPF, Fales HM (1988) Structural elucidation of alfalfa root saponins by mass spectrometry and nuclear magnetic resonance analysis. $J$ Chem Soc Perkin Trans 1, 3071-3079 
Monties B (1981) Les antinutritionnels. In: Protéines foliaires et alimentation ( $C$ Costes, éd), GauthierVillars, Paris, 93-120

Pietraszek W (1985) Contribution à l'étude des saponines chez la luzerne ( $M$ sativa $L$ ) : rôle antinutritionnel, variabilité génétique, aspects technologiques. Thèse docteur d'université, université ParisSud (Orsay), $68 p+$ annexes

Pracros P (1988a) Mesure de l'activité des saponines de la luzerne par les larves du ver de farine: Tenebrio molitor $L$ (coléoptère, Tenebrionidae). 1. Comparaison avec les résultats de divers tests biologiques, agronomie 8, 257-263

Pracros P (1988b) Mesure de l'activité des saponines de la luzerne par les larves du ver de farine:
Tenebrio molitor L (coléoptère, Tenebrionidae). 2. Recherche des fractions de saponines responsables des effets antinutritionnels observés. agronomie 8, 793-799

Rahbé Y, Febvay G, Delobel B, Bournoville R (1988) Acyrthosiphon pisum performance in response to the sugar and amino acid composition of artificial diets and its relation to lucerne varietal resistance. Entomol Exp App/ 48, 283-292

Wink M (1984) Biochemistry and chemical ecology of Lupin alkaloids. Actes $3^{e}$ Congrès international du lupin (La Rochelle, France), 326-343

Zimmer DE, Pedersen MW, McGuire CF (1967) A bioassay for alfalfa saponins using the fungus Trichoderma viride Pers. Crop Sci 7, 223-224 